\title{
The OSCs in the Crude Oil from the Jianghan Basin and their compositional variations during artificial secondary alterations
}

\author{
YuHONG LiAO ${ }^{1, *}$, WeIMIN LiU ${ }^{1}$, Bin JiANG ${ }^{1}$, PING’AN \\ $\mathrm{PENG}^{1}$ \\ ${ }^{1}$ State Key Laboratory of Organic Geochemistry, Guangzhou \\ Institute of Geochemistry, Chinese Academy of Sciences, \\ Guangzhou 510640, PR China
}

Organic sulfur compounds (OSCs) are abundant in some crude oils and a lot of species were reported in the immature crude oil in the Jianghan Basin. OScs in crude oils usually include both "unpolar" and "polar" OSCs. "Unpolar" OSCs refered to the compounds containing only sulfur atoms which were of low polarity and well studied by tradational instrumental technologies such as GC-MS. However, unpolar OSCs have not sufficient polarity to be analyzable by electrospray ionization (ESI) without derivatization. "Polar" OSCs are referred to the compounds containing one or more heteroatom(s) in addition to sulfur atoms which have high polarity and can be analyzed by electrospray ionization (ESI) without derivatization. Since FT-ICR MS can have ultra-high resolution and sensitivity, polar OSCs can be easily analyzed under negative mode without derivatization while unpolar OSCs can be analyzed under positive mode with derivatization (i.e., by the addition of $\mathrm{HCOONH}_{4}$ ). The development of ESI-FT-ICR MS provide a powerful tool for the full characterization of OSCs in crude oil. It is now possible to fully explore the origin of OSCs in crude oils and their secondary alteration mechanisms using ESI-FT-ICR MS In our work, we studied the OSCs in the crude oils from the Jianghan Basin and explored the intra-molecular sulfurization mechanisms by a Bruker SolariX XR 9.4T FT-ICR MS. The results indicated that sulfur incorporation (sulfurization) is usually abiotic and relied on the reactive functional groups such as carbon-carbon double bonds in hydrocarbons and carbohydrates. The detailful compositional variations in OSCs of the immature crude oil during secondary alterations such as aerobic biodegradation and thermal alteration, can also be monitored by ESI FT-ICR MS. The results indicated that micro-organisms preferentially attack alkyl chains in OSCs and generated the corresponding sulfur-containing acids. Terminal oxidation, rather than sulfur-specific degradation, is the more likely biodegradation pathway of OSCs. Additionally, in thermal simulation of crude oil, increasing aromatization of OSCs was observed by FT-ICR MS. 